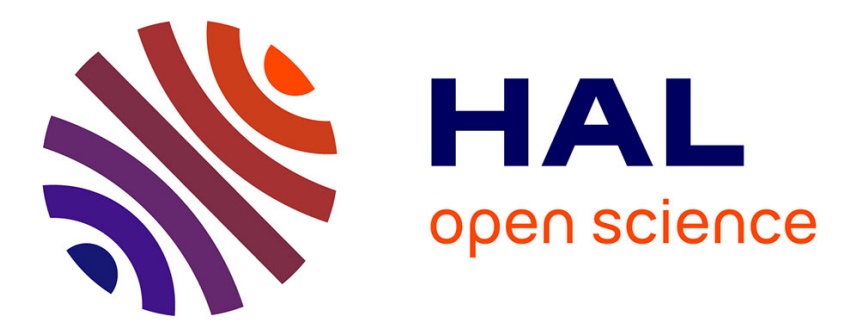

\title{
A new beam deflection angle amplification technique for mirage detection
}

\author{
A. Yarai, Y. Fukunaga, K. Sakamoto, T. Nakanishi
}

\section{To cite this version:}

A. Yarai, Y. Fukunaga, K. Sakamoto, T. Nakanishi. A new beam deflection angle amplification technique for mirage detection. Journal de Physique IV Proceedings, 1994, 04 (C7), pp.C7-79-C7-82. 10.1051/jp4:1994720 . jpa-00253248

\section{HAL Id: jpa-00253248 https://hal.science/jpa-00253248}

Submitted on 1 Jan 1994

HAL is a multi-disciplinary open access archive for the deposit and dissemination of scientific research documents, whether they are published or not. The documents may come from teaching and research institutions in France or abroad, or from public or private research centers.
L'archive ouverte pluridisciplinaire HAL, est destinée au dépôt et à la diffusion de documents scientifiques de niveau recherche, publiés ou non, émanant des établissements d'enseignement et de recherche français ou étrangers, des laboratoires publics ou privés. 


\title{
A new beam deflection angle amplification technique for mirage detection
}

\author{
A. Yarai, Y. Fukunaga, K. Sakamoto and T. Nakanishi \\ Department of Electrical Engineering and Electronics, Faculty of Engineering, Osaka Sangyo University, \\ Daito, Osaka, Japan
}

\begin{abstract}
A new technique has been developed for amplification of the photothermal beam deflection angle for mirage detection. This technique, based on a very simple operating principle, uses a cylindrical reflection mirror. The use of a new amplifier provided a signal-to-noise ratio approximately 10 times that obtained without the amplifier for equipment of the same size. By using the new amplifier, a mirage signal was obtained when a transistor array processed on a silicon wafer was measured.
\end{abstract}

\section{INTRODUCTION}

Mirage detection is a well-known non-destructive, non-contact and high-resolution detection method[1] [3]. The deflection angle, however, is very small, i.e. on the order of a few micro radians. Thus far, to amplify this small deflection angle, mainly $\mathrm{CCl}_{4}$ liquid has been used as the deflection medium[4][5]. $\mathrm{CCl}_{4}$ liquid, however, is known to be bitterly poisonous. Moreover, it has a large heat capacity compared with air, following that when an excitation beam is modulated at high frequency, the detected mirage signal is considerably decreased, and the reproducibility of measurement is worsened. Mirage detection with an excitation laser beam modulated at high frequency, however, is needed to analyze, for instance, depth profiles of samples.

This paper describes a new beam deflection angle amplification technique (an amplifier) for mirage detection. This new deflection angle amplifier's main part is composed of a cylindrical reflection mirror, which produces a very simple and compact structure. This technique solves the above-mentioned problems by increasing the signal-to-noise ratio even in small-sized equipment for mirage detection.

\section{FUNDAMENTAL AMPLIFICATION CHARACTERISTIC}

Figure 1 shows a block diagram of this new mirage detection setup containing a deflection angle amplifier. The principle [7] of this setup is as follows. When the probe laser beam strikes the cylindrical reflection mirror, the averaged reflected deflection beam angle is made larger (i.e. amplified) because of the cylindrical curvature of the mirror. This is not a problem, however, because the position of the energy center is detected on the position sensitive detector (PSD), if the beam has an approximate Gaussian beam profile.

Figure 2 shows the amplification characteristics, that is, the relationship between the incident deflection angle and the reflected deflection angle as a function of the cylindrical reflection mirror radius $r$. Here, the distance between the heated spot on the sample and the spot where the probe beam strikes the mirror is 70 $\mathrm{mm}$, and the angle $\theta$ (shown in the figure) is $45^{\circ}$. A carbon-black painted BK-7 glass substrate was used as the test sample. Input deflection angles were varied by varying the excitation laser power. As shown in the figure, even amplification of 120 times could be attained. However, the differences between the theoretical curves and the experimental data in the figure are relatively large when the mirror diameters are small. The main cause of this is considered to be the alignment errors in the setup. Nonetheless, it was 
found that the amplification linearity is maintained up to about a few milli-radians of incident deflection angles; it was experimentally proven. This linearity characteristic ${ }^{[7]}$ makes the setup sufficient for real use.

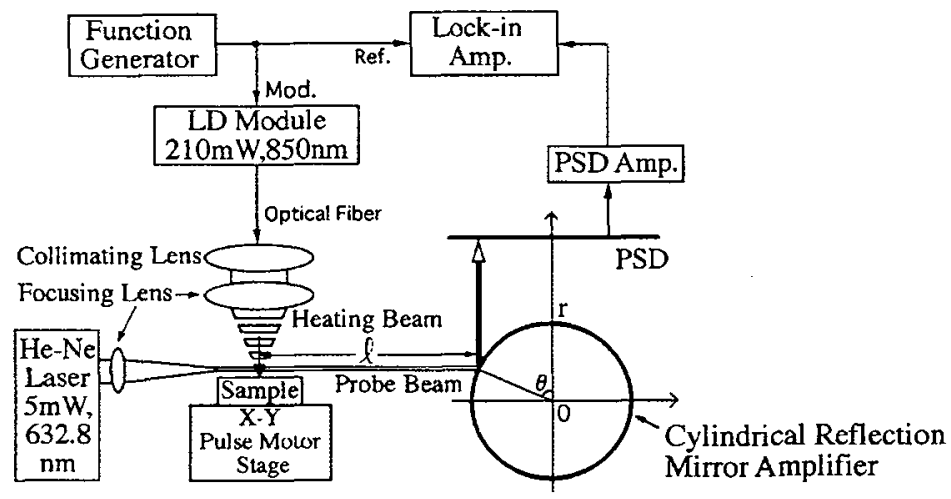

Fig.1. Block diagram of the new mirage setup

\section{SIGNAL-TO-NOISE RATIO ESTIMATE FOR THIS AMPLIFIER}

Figure 3 shows signal-to-noise ratio values obtained from measured data with and without the new amplifier in air. A high-power laser diode (fiber output power: $210 \mathrm{~mW}$, wavelength: 850 $\mathrm{nm}$ ) was used as an excitation heating source whose modulation frequency was adjusted at 1 $\mathrm{kHz}$. The transistor array sample mentioned above was also used as a test sample. Use of the amplifier provided a signal-to-noise ratio approximately 10 times that obtained without the amplifier, for a 120 $\mathrm{mm}$ distance between the beam deflection point on the test sample and the PSD. There are plural causes for noise when the signal is detected at the PSD. The reason why a signal-to-noise ratio could be improved is considered as follows. These include: 1) the fluctuation noise of a deflection beam angle, and 2) the electric noise of the PSD circuit. The former is amplified by our new amplifier, but the latter maintains a constant value. Additionally, the value of the latter is much larger than that of the former. Accordingly, the new amplifier shows an improvement in signal-to-noise ratio. This also explains why, in spite of the fact that our cylindrical mirror amplified the signal itself approximately 23 times on the PSD, the improvement in signal-to-noise ratio is only approximately 9 times $(26.4 / 2.93)$, as shown in Fig. 3 (when the "total distance" is $120 \mathrm{~mm}$ ). From these results, this amplification technique can be considered to be effective for mirage detection. Another important advantage is the compact setup size.

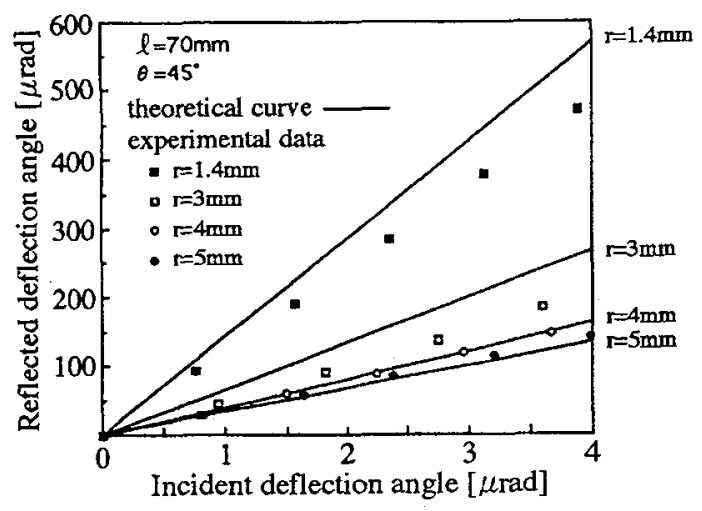

Fig.2. Relationship between the incident deflection angle and the reflected deflection angle

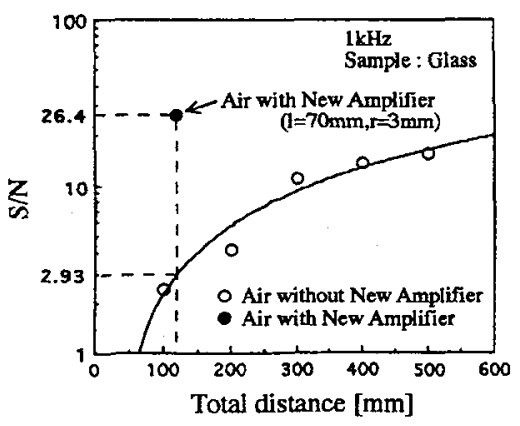

Fig.3. The total distance (between the beam deflection point on the sample and the point where the beam strikes the PSD) dependence of $\mathrm{S} / \mathrm{N}$ 
A transistor array processed on a silicon wafer was also used as a test sample to estimate the signal-tonoise ratio for this new technique. Figure 4 shows the sample structure and the scanning direction of the sample. Figure 5 shows the amplitude mirage signal obtained with this new method, as well as those obtained when $\mathrm{CCl}_{4}$ and air without the mirror were used as deflection media. It is obvious that when air is used as a reflection medium (i.e. a typical usage), the mirage signal distinctly shows the existence of transistors, both when the cylindrical reflection mirror is used and when it is not used. The mirage signal obtained by using this new amplifier, however, shows a higher amplitude compared with that without the amplifier. The signal for the $\mathrm{CCl}_{4}$ liquid unfortunately does not show the transistors. This is because of large noise resulting from 1) $\mathrm{CCl}_{4}$ liquid convection (when the heating laser is excited), and 2) liquid fluctuation (when the sample stage is being scanned).

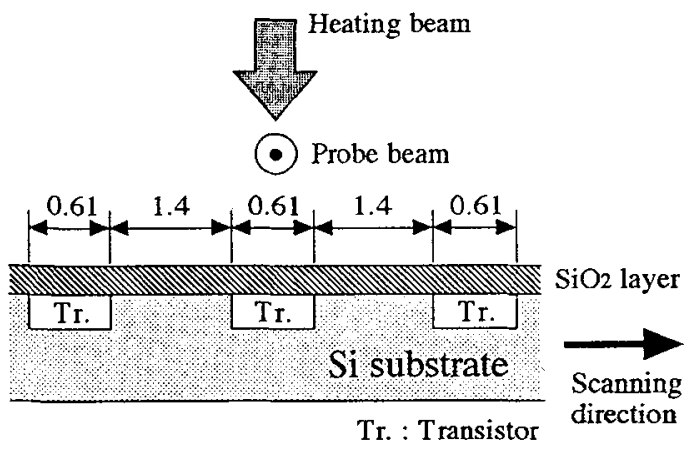

Fig.4. Sample structure (mm)

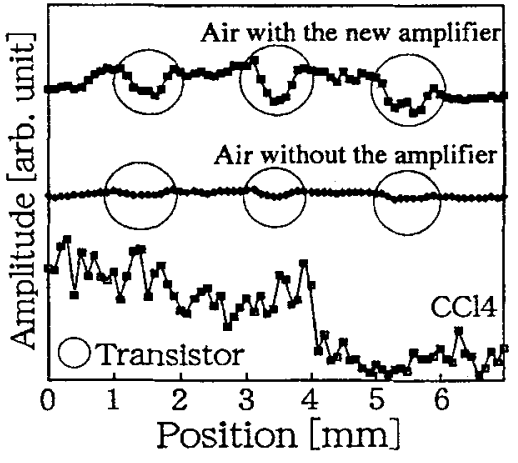

Fig. 5. Amplitude mirage signals (the origin of each curve is different.)

The deflection media are :

air with the new amplifier,

- air without the amplifier,

$\square \mathrm{CCl} 4$.
Here, the fluctuation amplitude for mirage signals was measured for three cases: air, water and $\mathrm{CCl}_{4}$ liquid being used as deflection media (Fig. 6). A 0.6-mm-thick Si wafer was used as the test sample. As shown in the figure, it was found that the mirage signal fluctuation amplitude, for $\mathrm{CCl}_{4}$ liquid, is more than approximately 40 times that of air. Therefore, the reason for the liquid fluctuation mentioned above was also demonstrated with this experimental result.

\section{FREQUENCY DEPENDENCE OF THE MIRAGE SIGNAL}

Figure 7 shows the modulation frequency dependence of the mirage signal for 3 cases: 1 ) use of the new amplifier, 2) use of $\mathrm{CCl}_{4}$ liquid and 3) absence of the amplification technique (i.e. use of the conventional method). As shown in the figure, though the $\mathrm{CCl}_{4}$ signal is high at low modulation frequencies, it decreases radically at high modulation frequencies. The reason for this is the larger heat capacity of $\mathrm{CCl}_{4}$ liquid compared to that of air. On the other hand, the mirage signal

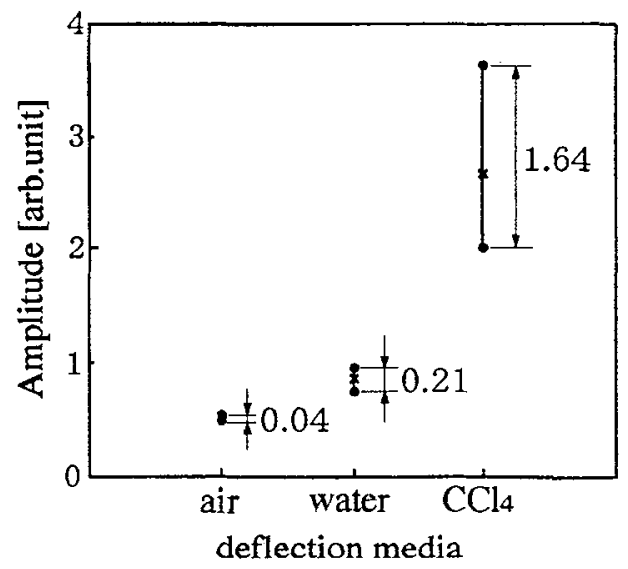

Fig.6. Fluctuation amplitude for mirage signals 
obtained with our deflection angle amplifier does not decrease significantly even at high modulation frequencies. Theoretical curves (ref. 6 was used for the theoretical calculations) also clearly confirmed the above tendencies. The differences between the theoretical curves and the experimental data, however, are relatively large. The reasons for this are assumed that, 1) the excitation laser diode beam is not completely absorbed into the test sample in the experiment, and 2) the beam profile is deviated from a Gaussian shape.

\section{CONCLUSION}

A new technique has been developed for the amplification of the photothermal beam deflection angle. This technique, based on a very simple operating principle, uses a cylindrical reflection mirror. Angle amplification of more than 120 times has been achieved. The use of a new amplifier provided a signal-to-noise ratio approximately 10 times that obtained without the amplifier for equipment of the same size. An important advantage, it should be noted, is the compactness of the setup size. Furthermore, the mirage signals obtained with this new deflection angle amplifier did not significantly decrease even in the high modulation frequency range. Therefore, this new beam deflection angle amplification technique for mirage detection can be considered to be an effective alternative for high-resolution imaging or high-sensitivity depth profile analysis of test samples.

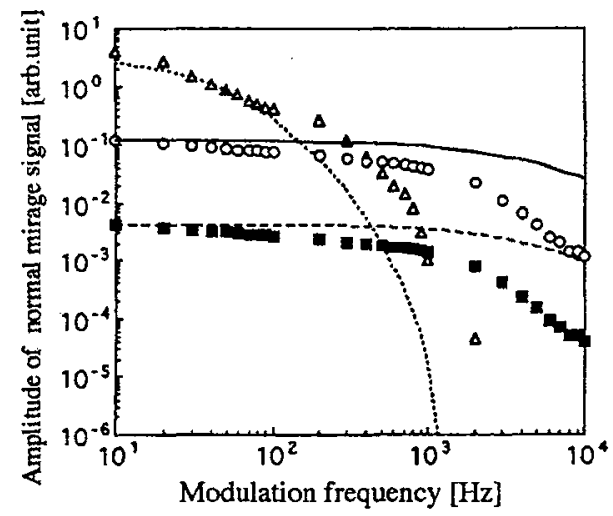

Fig.7. Modulation freqeuncy dependence of detection signal

The deflection media are :

$O$ air with the new amplifier

air without the new amplifier

$\triangle \mathrm{CCl} 4$

The theoretical curves are :

air with the new amplifier

- _ air without the new amplifier

-..-. CCl4

\section{References}

[1] A. C. Boccara, D. Fournier and J. Badoz, Appl. Phys. Lett. 36 (1980) 130.

[2] J. Rantala, J. Hartikainen and J. Jaarinen, Appl. Phys. A50 (1990) 465.

[3] K. Grice, L. Inglehart, L. D. Favro, P. K. Kuo and R. L. Thomas, J. Appl. Phys. 54 (1983) 6254.

[4] D. Fournier, Photoacoustic and Photothermal Phenomena III, (ed. D. Bicanic, Springer-Verlag, Heidelberg, 1991) 339.

[5] C. D. Bain and T. H. Ong, Photoacoustic and Photothermal Phenomena III, (ed. D. Bicanic, Springer-Verlag, Heidelberg, 1991) 158.

[6] A. Salazar, A. Sa nchez-Lavega and J. Ferna ndez, J. Appl. Phys. 65 (1989) 4150.

[7] A. Yarai, Y. Fukunaga, K. Sakamoto and T. Nakanishi, Jpn. J. Appl. Phys. 33 5B (1994), in printing. 